\title{
Evaluation of Th2 and Th17 Immunity-Related Factors as Indicators of Brucellosis
}

\section{OPEN ACCESS}

Edited by: Kamal El Bissati,

University of Chicago, United States

Reviewed by:

Jiabo Ding,

China Institute of Veterinary Drug

Control, China

Daniel Alford Powell,

University of Arizona, United States

Mariana Cristina Ferrero,

Consejo Nacional de Investigaciones

Científicas y Técnicas (CONICET),

Argentina

*Correspondence:

Reza Gheitasi

Reza.Gheitasi@med.uni-jena.de

Specialty section:

This article was submitted to

Clinical Microbiology,

a section of the journal

Frontiers in Cellular and

Infection Microbiology

Received: 30 September 2021

Accepted: 13 December 2021

Published: 07 January 2022

Citation:

Gheitasi R, Keramat F, Khosravi S,

Hajilooi M, Pletz MW and

Makarewicz O (2022) Evaluation of Th2

and Th17 Immunity-Related Factors

as Indicators of Brucellosis.

Front. Cell. Infect. Microbiol. 11:786994.

doi: 10.3389/fcimb.2021.786994
Reza Gheitasi ${ }^{1,2 *}$, Fariba Keramat ${ }^{3}$, Sara Khosravi ${ }^{4}$, Mehrdad Hajilooi $^{2}$, Mathias W. Pletz ${ }^{1}$ and Oliwia Makarewicz ${ }^{1}$

\footnotetext{
${ }^{1}$ Institute for Infectious Diseases and Infection Control, Jena University Hospital, Jena, Germany, ${ }^{2}$ Department of Immunology, School of Medicine, Hamadan University of Medical Sciences, Hamadan, Iran, ${ }^{3}$ Brucellosis Research Center, Hamadan University of Medical Sciences, Hamadan, Iran, ${ }^{4}$ Department of Microbiology, School of Medicine, Hamadan University of Medical Sciences, Hamadan, Iran
}

Objective: Brucellosis is a common bacterial zoonotic infection, and greater than half a million new cases are diagnosed annually. This study investigates the expression of Th2 and Th17 immunity-related factors (Th2-LCR IncRNA, IL-25, TRAF3IP2, and IL-17RB) in different stages of Brucella infections.

Material and Methods: In total, 99 brucellosis patients were divided into three groups (acute $=$ first infection before treatment, relapse $=$ before treatment, and treated $=$ after treatment for 6-8 weeks with doxycycline and rifampin). Thirty-three healthy volunteers represented the control group. Gene expression levels were assessed by quantitative amplification in reference to the 18S rRNA gene and statistically evaluated.

Results: No significant differences in the expression of these genes were observed between the control group and patients after completion of antibiotic treatment. Compared to these two groups, only Th2-LCR InCRNA and TRAF3IP2 were significantly more highly expressed in the acute group. Th2-LCR IncRNA was also significantly elevated in the relapse group. TRAF3IP2 expression was additionally significantly increased in the acute group compared to the relapse group.

Conclusion: IL-25 and IL-17RB failed to differentiate between the infected and noninfected groups. TRAF3IP2 and Th2-LCR IncRNA might be good indicators of brucellosis during the acute phase, but the expression levels varied strongly among patients. To verify the suitability of these factors as an indicator for brucellosis, acute infection or relapse should be investigated in further studies on larger cohorts with welldefined inclusion criteria.

Keywords: Brucella melitensis, adaptive immunity, cytokines, IncRNA, qPCR, arthralgias 


\section{INTRODUCTION}

With greater than half a million new cases per year (De Figueiredo et al., 2015), brucellosis is one of the most common zoonotic infectious diseases worldwide. Brucellosis is caused by Gram-negative, intracellular bacteria of the Brucella genus. Infections in both humans and animals typically occur by the oral or inhalation route via mucous membranes (Kaufmann et al., 1980; Dadar et al., 2019). Innate immune cells represent the first line of defense and try to eliminate invading pathogens. However, brucellae can overcome this defense by hiding and replicating in mononuclear cells (Rittig et al., 2001; Watarai et al., 2002), which spread the pathogen via the lymphatic system to various organs (Baldwin and Winter, 1994; Hasanjani Roushan et al., 2004). This condition manifests in a variety of nonspecific symptoms, varying from mildly acute to chronic (Rafiei et al., 2006), and the condition is even fatal in isolated cases (Carrington et al., 2012).

To date, known intracellular signaling cascades in brucellosis have been described in detail in several reviews elsewhere (De Figueiredo et al., 2015; López-Santiago et al., 2019; Ferrero et al., 2020). Briefly, innate antigen-presenting cells (APCs) activate naïve $\mathrm{CD}^{+} \mathrm{T}$ helper $(\mathrm{Th})$ cells and $\mathrm{CD} 8^{+}$cytotoxic $\mathrm{T}(\mathrm{Tc})$ cells. The primed $\mathrm{CD} 4^{+}$Th cells further differentiate into Th1 cells or Th2 cells in response to the specific cytokine milieu provided by activated APCs. Activated macrophages and Th1 cells control Brucella infection mainly by IFN $\gamma$ secretion, which further stimulates the cellular response (Giambartolomei et al., 2002; Brandão et al., 2012) via $\mathrm{CD}^{+}$cytotoxic T cells of type 1 (Tc1) against Brucella-infected cells (Skendros and Boura, 2013; Rahmanpour et al., 2019). During the progression of infection, humoral immunity increases upon stimulation by IL-4producing $\mathrm{CD}^{+}$Th2 cells, which mediate the activation of $\mathrm{B}$ cells and subsequent antibody production (Zhu, 2015). Natural killer (NK) cells also appear to play an important role in the pathogenesis of brucellosis through the early production of IFN $\gamma$ and their cytotoxicity, which has been shown to be inhibited during acute brucellosis (Salmerón et al., 1992). NK cells may also induce antibody production by B cells (Gao et al., 2011).

During intracellular infections, Th17-mediated immunity (often associated with autoimmune diseases) has also been shown to be activated, bridging and modulating both the Th1 and Th2 responses by IL-25 (also known as IL-17E) (Bai et al., 2009). IL-25 is produced by damaged epithelial cells or activated mast cells, macrophages, eosinophils, and Th2 cells and is mainly associated with allergic diseases (Besnard et al., 2011). IL-25 receptors (composed of IL-17RA and IL-17RB subunits) are present in both NK cells and $\mathrm{CD} 4{ }^{+}$Th cells (Terashima et al., 2008; Stock et al., 2009). The signaling cascades initiated from the receptors of the IL-17 family are mediated by TRAF3 interacting protein 2 (TRAF3IP2, also known as Act1), a key upstream activator of various inflammatory mediators. IL-25 can suppress Th1 and Th17 immune responses by inhibiting IL-12 or inducing IL-23 production, respectively, reducing the tissuedamaging effects of inflammation (Caruso et al., 2009). IL-25 also increases the secretion of Th2-related cytokines (including IL-4), stimulating the humoral response. IL-4 is encoded in one gene cluster that includes genes for IL-5 and IL-13 and a locus control region (LCR) (Chang et al., 2006) that regulates the expression of these cytokines (Zeng, 2013). The LCR overlaps a long noncoding (lnc)RNA (hereafter referred to as Th2-LCRlncRNA) that is predominantly expressed in primary and effector Th2 cells (Spurlock et al., 2015) and positively regulates the transcription of IL-4, IL-5, and IL-13. The Th2-LCR IncRNA profile can be altered by pathogens, thereby influencing the immune response and pathogenicity (Wen et al., 2020).

We investigated the expression of IL-25, IL-17RB, Th2-LCR IncRNA, and TRAF3IP2, which play roles in brucellosis. The aim of this study was to assess the potential of these Th2 and Th17 factors as markers for the infection status defined as acute or relapse. To the best of our knowledge, this study represents the first preliminary data on TRAF3IP2 and Th2-LCR IncRNA expression in humans suffering from brucellosis.

\section{MATERIALS AND METHODS}

\section{Ethical Approval and Patient Consent}

This study was approved by the local ethical committee of the Hamadan University of Medical Sciences under reference number IR.UMSHA.REC.1397.760. All participating patients and volunteers were informed about the study, applied procedures and any risks due to sampling by a physician and provided their written consent. Participants were also informed that they could withdraw from the study at any time.

\section{Sample Collection and RNA Extraction}

Blood samples were collected in $10-\mathrm{ml}$ vacutainer tubes containing EDTA (BD Vacutainer, USA) from patients and volunteers upon written consent between July 2018 and April 2019. Total RNA was extracted using RNX-plus solution (CinnaGene, Iran, RN7713C) following the manufacturer's instructions. The concentration and purity of extracted RNA were analyzed using a Nanodrop (A\&E Lab, Nano200, UK), and the integrity of total RNA was controlled by gel electrophoresis in a $1 \%$ agarose gel supplemented with $2.2 \mathrm{M}$ formaldehyde in MOPS-buffer.

\section{Reverse Transcription Reaction and qPCR} Primers (Supplementary Material, Table S2) were designed for the LCR-IncRNA, IL-17E, IL17-RB, TRAF3IP2, and 18S rRNA genes. The primers were provided by the Bioneer company (South Korea) in lyophilized form and resuspended in DEPC-treated water to a final concentration of $10 \mu \mathrm{M}$ and stored at $-20^{\circ} \mathrm{C}$.

cDNA synthesis and quantitative PCR (qPCR) were performed using $1 \mu \mathrm{g}$ of total RNA as previously described in triplicate (Gheitasi et al., 2020). Signals reaching the threshold within 36 cycles were considered for quantification. The $\triangle \mathrm{Ct}$ values of each target were assessed in relation to $18 \mathrm{~S}-\mathrm{rRNA}$. The qPCR efficiency for all primer pairs was $\varnothing 2.01 \pm 0.05$ (between 1.97 and 2.0) and thus almost identical. Therefore, the relative fold change in transcripts of the targets was calculated based on the $2^{-\triangle \triangle \mathrm{Ct}}$ in relation to the mean of the control group for all individual patients (including control group). 


\section{Statistical Analysis}

GraphPad Prism version 8 (GraphPad Software, USA) was applied for all statistical analyses. The normality of variables was assessed using the Shapiro-Wilk test. To compare the groups, two-way ANOVA with Bonferroni's multiple comparison test was performed for the $2^{-\triangle \triangle \mathrm{Ct}}$ values. A $P$ value less than 0.05 was considered significant. Correlations between all pairs of datasets were analyzed using Pearson's rank test.

\section{RESULTS}

\section{Study Population and Clinical Features}

Patients referred to the Infectious Diseases Centre of Sina Hospital (Hamadan, Iran) with suspected brucellosis who showed clinical manifestations, such as fever $\left(<37^{\circ} \mathrm{C}\right)$ or chills, subjective weight loss, headache, and body pain (namely, myalgia, bone and/or joint pain), were eligible for the patient groups. Subjective manifestations were collected using a questionnaire. The diagnosis of brucellosis was confirmed by serological tests, including the Wright test (titer $\geq 1 / 80$ ) and 2-mercaptoethanol (2-ME) test (titer $\geq 1 / 40$ ), and approved by infectious disease specialists. In total, 99 patients (67.67\% male and $32.32 \%$ female) with brucellosis were enrolled into three patient subgroups (each of 33 patients) that were defined as follows: I) Acute: patients with positive serological tests and related symptoms for the first time and before treatment. II) Treatment: patients with symptoms and clinical manifestations directly after they received routine treatment $(100 \mathrm{mg} / \mathrm{BD}$ doxycycline and $600 \mathrm{mg} /$ day rifampin) for four to eight weeks for the first time. III) Relapse: patients who displayed clinical symptoms between three months and two years after first routine treatment and not under treatment with a second therapy to date. Additionally, a control group of 30 individuals selected based on negative serological tests and history of other infectious diseases was included. Pregnant women and patients with other infectious diseases, cancers or autoimmune diseases were excluded.

The descriptive analysis of this study cohort (Supplementary Material, Table S1) was already published in a previous work, where other markers (cMAF and Linc-MAF-4) were investigated (Gheitasi et al., 2020). Briefly summarizing, within the patient subgroups, the sex and age distribution were similar with twice as many male cases and an average age of $45.96 \pm 15.36$ years. In the control group, gender was equally distributed, and the average age was slightly lower at $41.03 \pm 9.48$. According to clinical characteristics, most patients suffer from fever, chills and arthralgias $(\sim 72.1 \%)$. Other clinical manifestations exhibited among the study population included the following: weight loss $(31.3 \%)$, headache $(46.4 \%)$, and body pain and myalgia (approximately $47.4 \%$ ).

\section{Increased TRAF3IP2 and Th2-LCR Expression in Infected Patients}

The expression level of the 18S rRNA gene ranked between $10.04 \pm$ 1.858 and $11.02 \pm 1.515$ cycles (means and SD), passed the normality test, and did not differ significantly between the groups $(\mathrm{P}=0.491)$ (Supplementary Material, Table S3 and Figure S1A), indicating its suitability as a reference gene in this experimental setup.

In general, the $\triangle \mathrm{Ct}$ values of the chosen 4 target genes varied more strongly than the expression of 18S-rRNA (Supplementary Material, Figure S1B), and some targets in some groups (IL-25 and Th2-LCR lncRNA in the relapse group and IL-17RB and TRAF3IP2 in the acute group) did not pass the normality test (Supplementary Material, Table S3, indicated in red). In the control group, the distribution of the $\triangle \mathrm{Ct}$ values passed the normality test for all target genes.

The control group was used to normalize and compare the changes in the expression levels of the targets between the groups. The mean $\triangle \mathrm{Ct}$ value of the control groups was used for this purpose and was subtracted from all individual values, including the control group values, to visualize the variation within this group. With a percentage coefficient of variation (\% $\mathrm{CV}$ ) of $>100$, the standard deviation was relatively high in all groups (Supplementary Material, Table S4). However, the $2^{-\triangle \triangle \mathrm{Ct}}$ of the control group was less than 20 and showed a similar pattern for all target genes (Figure 1). The expression patterns of IL-25 and IL-17RB did not significantly differ in the groups (Figure 1). Despite the high scattering of values, the expression of TRAF3IP2 and Th2-LCR lncRNA was significantly increased in the acute group, and Th2-LCR lncRNA was additionally increased in the relapse group. Compared to each other, neither expression pattern differed significantly.

\section{DISCUSSION}

Most studies on the immune response and modulation due to Brucella infection have been performed in cell-line models and animals, and the pathophysiology of this condition in humans is still not fully understood. The present study aimed to investigate specific immune factors in patients suffering from brucellosis in the acute and release phases (before antibiosis) and also in patients not receiving antibiotic treatment and to compare the expression of these factors in relation to the healthy control group.

From a different cohort from the Sina Hospital collected a year prior the present study, it was shown that Th1 and Th17 cell subsets were increased in the acute and relapse patients compared to the other two groups (Rahmanpour et al., 2019). The increased concentrations of IFN- $\gamma$ in the acute and relapse groups were in agreement with the elevated Th1 cell content, but no significant increase was observed for the IL-17A that is secreted by activated Th17 cells. Simultaneously, the IL-22, as a cytokine related to defense of epithelial cells against invading pathogens was increased in acute and relapse patients providing some evidence for protective immunity against Brucella. Another recent study for a brucellosis cohort from China also showed increased levels of IL-17A in acute (defined as duration of disease was less than 6 months) and chronic infections (defined as not recovered for more than 6 month), but in contrast, Th17 cell counts was not significantly elevated (Zheng et al., 2019). In that 


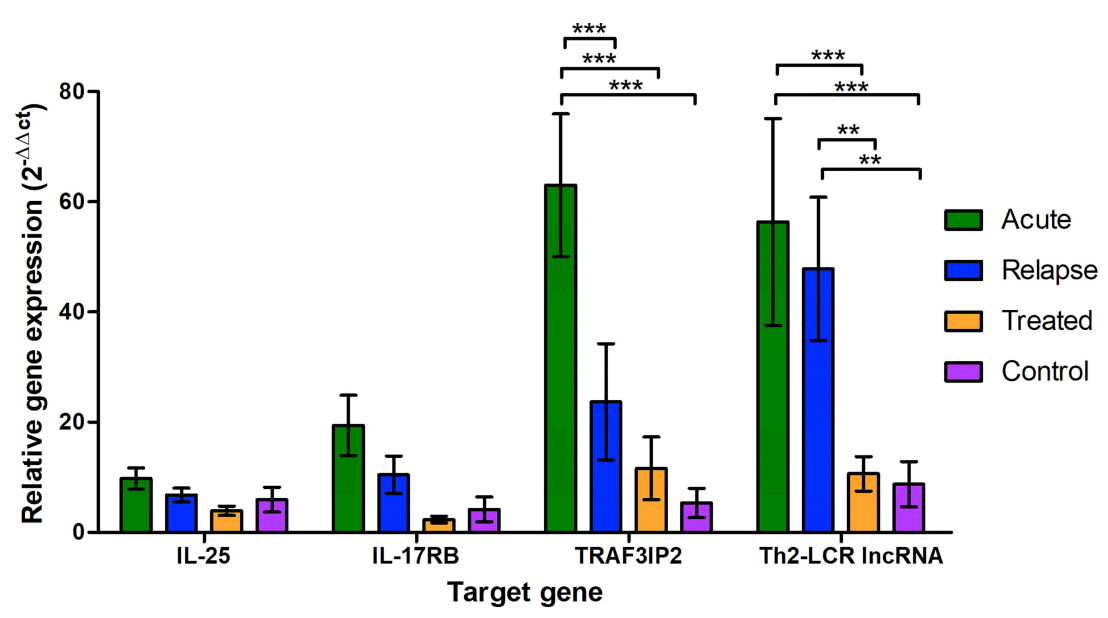

FIGURE 1 | Relative expression of the selected genes (X-axis) in the different study groups (legend) shown as $2^{-\triangle \triangle C t}$ to the reference gene (18S-rRNA) and the mean of the control group. The values are presented as the mean and the standard error of the mean $(\mathrm{SEM}){ }^{*} \mathrm{P}<0.05$, ${ }^{* *} \mathrm{P} \leq 0.01$, and ${ }^{* \star *} \mathrm{P}<0.001$.

study, the Th2 cell count and also IL-5, Il-4, and Il-13 were particularly elevated in chronic patients. In a previous study on the present cohort (Gheitasi et al., 2020), the expression of cMAF, a transcriptional activation e.g., of IL-10 related with the shift towards Th2 response (Cao et al., 2005), was significantly higher in the relapse group when compared to the healthy controls. These previous studies lead us to the hypothesis that there is a shift to a protective autoimmunity and humoral immune response in brucellosis. Thus, this work focused on the expression of four Th2 and Th17 immunity-related factors (Th2LCR IncRNA, IL-25, TRAF3IP2, and IL-17RB) in different stages of brucellosis.

The current study revealed that TRAF3IP2 and Th2-LCR lncRNA expression was particularly increased in the acute phase of brucellosis. IL-25 and IL-17RB apparently failed to differentiate between the infected and noninfected groups. During relapse, TRAF3IP2 expression seemed to decrease, whereas the Th2-LCR lncRNA level remained high.

Th2-LCR lncRNA is required for the expression of IL-4, IL-5 and Il-13 and subsequently for $\mathrm{CD}^{+}$Th-cell differentiation and humoral response (Spurlock et al., 2015). The expression of Th2LCR IncRNA has thus far not been investigated in brucellosis or other bacterial infections, and it is unclear to what extent this factor is selective for brucellosis.

TRAF3IP2 is the key activator of Th17-mediated inflammatory responses but also suppresses the humoral B cell response by negatively regulating $\mathrm{CD} 40 \mathrm{~L}$ and $\mathrm{BAFF}$ signaling (Qian et al., 2004). TRAF3IP2 seems to be more specific to Brucella infections than other Gram-negative bacteria. In the study of Degos et al., TRAF3IP2 expression was increased fourfold in human blood DCs after exposure to the Brucella virulence factor $C \beta G$ ( $\beta-1,2$ cyclic glucan) compared to the E. coli lipopolysaccharides (LPS) (Degos et al., 2015) C $\beta G$ is a highly abundant virulence factor of Brucella that modulates membrane rafts of the infected cell necessary for the intracellular escape
(Arellano-Reynoso et al., 2005). It is thus one of the most Brucella-specific toxins recognized by immune cells (ArellanoReynoso et al., 2005; Degos et al., 2015). LPS is another important Gram-negative virulence factor. However, it is altered in Brucella, so it is only weakly immunostimulatory (Lapaque et al., 2005; Barquero-Calvo et al., 2007).

TRAF3IP2 might represent an interesting candidate as a potent marker that might even be useful to differentiate between patients with acute and relapsed brucellosis. However, this differentiation would require the use of an additional, wellsuited reference gene from the immune cascade for normalization (e.g., based on a ratio), as the $\Delta \mathrm{Ct}$ alone fluctuated too much. In addition, comparisons with other nonbrucellosis-infected groups should be performed to test for selectivity. It is possible that this factor has a more general function in specific infections (e.g., intracellular bacteria or cell damage).

The wide range of the qPCR results generally points to fundamental difficulties in quantitative analyses of patient material. Immunological parameters are difficult to differentiate cleanly, as the immune response is influenced by many individual factors that are generally difficult to define and record in the study design. The observed non-Gaussian distribution of the acute and relapse groups might result from unreliable assignment to the groups. Given that the classification 'acute' was based on a subjective survey of the patients, patients may have given false information against their better knowledge. For the classification 'relapse', the different characteristics of pathogenesis and chronification could have played roles. Therefore, in further investigations of brucellosis in patients, the inclusion criteria and group assignments should be more clearly and tightly defined. For example, it could help to include only patients whose medical history is well documented and to use standardized questionnaires that are also completed by hospital staff based on medical history and documentation. Longitudinal prospective studies, which are 
admittedly more difficult to conduct, would be excellent for assessing the changes in specific factors or markers in the individual course of the disease to better understand the immunological processes in brucellosis.

\section{DATA AVAILABILITY STATEMENT}

The original contributions presented in the study are included in the article/Supplementary Material. Further inquiries can be directed to the corresponding author.

\section{ETHICS STATEMENT}

The studies involving human participants were reviewed and approved by the Iran National Committee for Ethics in Biomedical Research, Hamadan University of Medical Sciences (IR.UMSHA.REC.1397.760). The patients/participants provided their written informed consent to participate in this study.

\section{AUTHOR CONTRIBUTIONS}

Conceptualization: FK and MH. Methodology: MH. and RG. Investigation: RG. Validation: FK, MH, and RG. Formal analysis: OM and RG. Resources: MH, RG, and MP. Data curation: OM and RG. Writing-Original draft preparation: SK and RG.

\section{REFERENCES}

Arellano-Reynoso, B., Lapaque, N., Salcedo, S., Briones, G., Ciocchini, A. E., Ugalde, R., et al. (2005). Cyclic Beta-1,2-Glucan Is a Brucella Virulence Factor Required for Intracellular Survival. Nat. Immunol. 6, 618-625. doi: 10.1038/ni1202

Bai, H., Cheng, J., Gao, X., Joyee, A. G., Fan, Y., Wang, S., et al. (2009). IL-17/Th17 Promotes Type $1 \mathrm{~T}$ Cell Immunity Against Pulmonary Intracellular Bacterial Infection Through Modulating Dendritic Cell Function. J. Immunol. 183, 5886-5895. doi: 10.4049/jimmunol.0901584

Baldwin, C. L., and Winter, A. J. (1994). Macrophages and Brucella. Immunol. Ser. $60,363-380$

Barquero-Calvo, E., Chaves-Olarte, E., Weiss, D. S., Guzmán-Verri, C., ChacónDíaz, C., Rucavado, A., et al. (2007). Brucella Abortus Uses a Stealthy Strategy to Avoid Activation of the Innate Immune System During the Onset of Infection. PloS One 2, e631. doi: 10.1371/journal.pone.0000631

Besnard, A. G., Sabat, R., Dumoutier, L., Renauld, J. C., Willart, M., Lambrecht, B., et al. (2011). Dual Role of IL-22 in Allergic Airway Inflammation and its CrossTalk With IL-17a. Am. J. Respir. Crit. Care Med. 183, 1153-1163. doi: 10.1164/ rccm.201008-1383OC

Brandão, A. P., Oliveira, F. S., Carvalho, N. B., Vieira, L. Q., Azevedo, V., Macedo, G. C., et al. (2012). Host Susceptibility to Brucella Abortus Infection is More Pronounced in IFN- $\gamma$ Knockout Than IL-12/ 32 -Microglobulin DoubleDeficient Mice. Clin. Dev. Immunol. 2012, 589494. doi: 10.1155/2012/589494

Cao, S., Liu, J., Song, L., and Ma, X. (2005). The Protooncogene C-Maf Is an Essential Transcription Factor for IL-10 Gene Expression in Macrophages. J. Immunol. 174, 3484-3492. doi: 10.4049/jimmunol.174.6.3484

Carrington, M., Choe, U., Ubillos, S., Stanek, D., Campbell, M., Wansbrough, L., et al. (2012). Fatal Case of Brucellosis Misdiagnosed in Early Stages of Brucella Suis Infection in a 46-Year-Old Patient With Marfan Syndrome. J. Clin. Microbiol. 50, 2173-2175. doi: 10.1128/JCM.00573-12

Caruso, R., Sarra, M., Stolfi, C., Rizzo, A., Fina, D., Fantini, M. C., et al. (2009). Interleukin-25 Inhibits Interleukin-12 Production and Th1 Cell-Driven
Writing-Review and editing: OM, RG, and MP. Visualization: $\mathrm{OM}$ and RG. Supervision and project administration: FK and $\mathrm{MH}$. All authors contributed to the article and approved the submitted version.

\section{FUNDING}

This work was supported by grants from the Vice Chancellor for Research and Technology at Hamadan University of Medical Sciences under the grant number 9710186141 and from the European Union's Horizon 2020 research and innovation program under Marie Skłodowska-Curie grant agreement number 861323.

\section{ACKNOWLEDGMENTS}

We would like to thank the medical and nursing staff of the Sina Hospital for their support in sample collection and Sebastian Drube for his critical reading and comments on the manuscript.

\section{SUPPLEMENTARY MATERIAL}

The Supplementary Material for this article can be found online at: https://www.frontiersin.org/articles/10.3389/fcimb.2021. 786994/full\#supplementary-material

Inflammation in the Gut. Gastroenterology 136, 2270-2279. doi: 10.1053/ j.gastro.2009.02.049

Chang, S. H., Park, H., and Dong, C. (2006). Act1 Adaptor Protein is an Immediate and Essential Signaling Component of Interleukin-17 Receptor. J. Biol. Chem. 281, 35603-35607. doi: 10.1074/jbc.C600256200

Dadar, M., Shahali, Y., and Whatmore, A. M. (2019). Human Brucellosis Caused by Raw Dairy Products: A Review on the Occurrence, Major Risk Factors and Prevention. Int. J. Food Microbiol. 292, 39-47. doi: 10.1016/ j.ijfoodmicro.2018.12.009

De Figueiredo, P., Ficht, T. A., Rice-Ficht, A., Rossetti, C. A., and Adams, L. G. (2015). Pathogenesis and Immunobiology of Brucellosis: Review of BrucellaHost Interactions. Am. J. Pathol. 185, 1505-1517. doi: 10.1016/ j.ajpath.2015.03.003

Degos, C., Gagnaire, A., Banchereau, R., Moriyon, I., and Gorvel, J. P. (2015). Brucella CbetaG Induces a Dual Pro- and Anti-Inflammatory Response Leading to a Transient Neutrophil Recruitment. Virulence 6, 19-28. doi: 10.4161/21505594.2014.979692

Ferrero, M. C., Alonso Paiva, I. M., Munoz Gonzalez, F., and Baldi, P. C. (2020). Pathogenesis and Immune Response in Brucella Infection Acquired by the Respiratory Route. Microbes Infect. 22, 407-415. doi: 10.1016/ j.micinf.2020.06.001

Gao, N., Jennings, P., Guo, Y., and Yuan, D. (2011). Regulatory Role of Natural Killer (NK) Cells on Antibody Responses to Brucella Abortus. Innate Immun. 17, 152-163. doi: 10.1177/1753425910367526

Gheitasi, R., Keramat, F., Solgi, G., and Hajilooi, M. (2020). Investigation of LincMAF-4 Expression as an Effective Marker in Brucellosis. Mol. Immunol. 123, 60-63. doi: 10.1016/j.molimm.2020.04.022

Giambartolomei, G. H., Delpino, M. V., Cahanovich, M. E., Wallach, J. C., Baldi, P. C., Velikovsky, C. A., et al. (2002). Diminished Production of T Helper 1 Cytokines Correlates With T Cell Unresponsiveness to Brucella Cytoplasmic Proteins in Chronic Human Brucellosis. J. Infect. Dis. 186, 252-259. doi: $10.1086 / 341449$ 
Hasanjani Roushan, M. R., Mohrez, M., Smailnejad Gangi, S. M., Soleimani Amiri, M. J., and Hajiahmadi, M. (2004). Epidemiological Features and Clinical Manifestations in 469 Adult Patients With Brucellosis in Babol, Northern Iran. Epidemiol. Infect. 132, 1109-1114. doi: 10.1017/S0950268804002833

Kaufmann, A. F., Fox, M. D., Boyce, J. M., Anderson, D. C., Potter, M. E., Martone, W. J., et al. (1980). Airborne Spread of Brucellosis. Ann. N. Y. Acad. Sci. 353, 105-114. doi: 10.1111/j.1749-6632.1980.tb18912.x

Lapaque, N., Moriyon, I., Moreno, E., and Gorvel, J. P. (2005). Brucella Lipopolysaccharide Acts as a Virulence Factor. Curr. Opin. Microbiol. 8, 6066. doi: 10.1016/j.mib.2004.12.003

López-Santiago, R., Sánchez-Argáez, A. B., De Alba-Núñez, L. G., Baltierra-Uribe, S. L., and Moreno-Lafont, M. C. (2019). Immune Response to Mucosal Brucella Infection. Front. Immunol. 10, 1759-1759. doi: 10.3389/fimmu.2019.01759

Qian, Y., Qin, J., Cui, G., Naramura, M., Snow, E. C., Ware, C. F., et al. (2004). Act1, A Negative Regulator in CD40- and BAFF-Mediated B Cell Survival. Immunity 21, 575-587. doi: 10.1016/j.immuni.2004.09.001

Rafiei, A., Ardestani, S. K., Kariminia, A., Keyhani, A., Mohraz, M., and Amirkhani, A. (2006). Dominant Th1 Cytokine Production in Early Onset of Human Brucellosis Followed by Switching Towards Th2 Along Prolongation of Disease. J. Infect. 53, 315-324. doi: 10.1016/j.jinf.2005.11.024

Rahmanpour, M., Keramat, F., Jourghasemi, S., Rashidi, G., Abdolmaleki, M., Solgi, G., et al. (2019). Direct Correlation Between Th1 and Th17 Responses in Immunity to Brucella Infection. Microbes Infect. 21, 441-448. doi: 10.1016/ j.micinf.2019.05.002

Rittig, M. G., Alvarez-Martinez, M. T., Porte, F., Liautard, J. P., and Rouot, B. (2001). Intracellular Survival of Brucella Spp. In Human Monocytes Involves Conventional Uptake But Special Phagosomes. Infect. Immun. 69, 3995-4006. doi: 10.1128/IAI.69.6.3995-4006.2001

Salmerón, I., Rodríguez-Zapata, M., Salmerón, O., Manzano, L., Vaquer, S., and Alvarez-Mon, M. (1992). Impaired Activity of Natural Killer Cells in Patients With Acute Brucellosis. Clin. Infect. Dis. 15, 764-770. doi: 10.1093/clind/ 15.5.764

Skendros, P., and Boura, P. (2013). Immunity to Brucellosis. Rev. Sci. Tech. 32, 137-147. doi: 10.20506/rst.32.1.2190

Spurlock, C. F.3rd, Tossberg, J. T., Guo, Y., Collier, S. P., Crooke, P. S.3rd, and Aune, T. M. (2015). Expression and Functions of Long Noncoding RNAs During Human T Helper Cell Differentiation. Nat. Commun. 6, 6932. doi: 10.1038/ncomms7932

Stock, P., Lombardi, V., Kohlrautz, V., and Akbari, O. (2009). Induction of Airway Hyperreactivity by IL-25 Is Dependent on a Subset of Invariant NKT Cells
Expressing IL-17rb. J. Immunol. 182, 5116-5122. doi: 10.4049/ jimmunol.0804213

Terashima, A., Watarai, H., Inoue, S., Sekine, E., Nakagawa, R., Hase, K., et al. (2008). A Novel Subset of Mouse NKT Cells Bearing the IL-17 Receptor B Responds to IL-25 and Contributes to Airway Hyperreactivity. J. Exp. Med. 205, 2727-2733. doi: 10.1084/jem.20080698

Watarai, M., Makino, S., Fujii, Y., Okamoto, K., and Shirahata, T. (2002). Modulation of Brucella-Induced Macropinocytosis by Lipid Rafts Mediates Intracellular Replication. Cell Microbiol. 4, 341-355. doi: 10.1046/j.14625822.2002.00195.x

Wen, Y., Chen, H., Luo, F., Zhou, H., and Li, Z. (2020). Roles of Long Noncoding RNAs in Bacterial Infection. Life Sci. 263, 118579. doi: 10.1016/ j.lfs.2020.118579

Zeng, W. P. (2013). All Things Considered: Transcriptional Regulation of T Helper Type 2 Cell Differentiation From Precursor to Effector Activation. Immunology 140, 31-38. doi: 10.1111/imm.12121

Zheng, R., Xie, S., Zhang, Q., Cao, L., Niyazi, S., Lu, X., et al. (2019). Circulating Th1, Th2, Th17, Treg, and PD-1 Levels in Patients With Brucellosis. J. Immunol. Res. 2019, 3783209. doi: 10.1155/2019/3783209

Zhu, J. (2015). T Helper 2 (Th2) Cell Differentiation, Type 2 Innate Lymphoid Cell (ILC2) Development and Regulation of Interleukin-4 (IL-4) and IL-13 Production. Cytokine 75, 14-24. doi: 10.1016/j.cyto.2015.05.010

Conflict of Interest: The authors declare that the research was conducted in the absence of any commercial or financial relationships that could be construed as a potential conflict of interest.

Publisher's Note: All claims expressed in this article are solely those of the authors and do not necessarily represent those of their affiliated organizations, or those of the publisher, the editors and the reviewers. Any product that may be evaluated in this article, or claim that may be made by its manufacturer, is not guaranteed or endorsed by the publisher.

Copyright (C) 2022 Gheitasi, Keramat, Khosravi, Hajilooi, Pletz and Makarewicz. This is an open-access article distributed under the terms of the Creative Commons Attribution License (CC BY). The use, distribution or reproduction in other forums is permitted, provided the original author(s) and the copyright owner(s) are credited and that the original publication in this journal is cited, in accordance with accepted academic practice. No use, distribution or reproduction is permitted which does not comply with these terms. 